\title{
Dinofurcula cf. ventralis en la costa central del Perú y primeros registros de dos especies de Protoperidinium
}

\section{Dinofurcula cf. ventralis in the central coast of Peru and the first report of two Protoperidinium species}

\author{
Noemí Ochoa' ${ }^{1}$ y Maribel Baylón
}

Presentado: 21/10/2005

Aceptado: 06/01/2006

\section{Resumen}

El presente trabajo informa de la presencia de Dinofurcula cf. ventralis, un dinoflagelado extremadamente raro que no ha sido registrado para el Pacífico peruano desde su descripción original. También se informa de dos primeros registros para el Perú, Protoperidinium thulesense y Protoperidinium paulseni. Las observaciones procedieron de las muestras colectadas periódicamente entre 1992 y 2004, en una

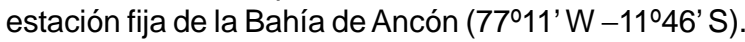

Palabras clave: Dinofurcula, Protoperidinium thulesense, P. paulseni, Bahía de Ancón, Perú.

\section{Abstract}

This paper report Dinofurcula cf. ventralis, a very rare dinoflagellate that has not been recorded for Peruvian Pacific since their original description. Also, it reports Protoperidinium thulesense and Protoperidinium paulseni for the first time in Peru. The observations proceeded of the samples collected periodically between 1992-2004 in the Ancón Bay (7711' W -11ㄴ6' S)

Keywords: Dinofurcula, Protoperidinium thulesense, P. paulseni, Ancón Bay, Peru.

\section{Introducción}

Los dinoflagelados constituyen el segundo grupo de mayor diversidad de especies del fitoplancton marino. En 1999 Ochoa et al. reportaron 208 especies de dinoflagelados marinos para el Perú, desde esa fecha el número de dinoflagelados se ha incrementado cada cierto tiempo con nuevos registros.

En este trabajo presentamos observaciones morfológicas de Dinofurcula cf. ventralis, y de las especies Protoperidinium thulesense Balech 1973 y P. paulseni (Abe 1981), tres dinoflagelados encontrados en la Bahía de Ancón, Lima, Perú.

El género Dinofurcula, fue establecido en 1928 por Kofoid y Skogsberg, a partir de material colectado en 1907 en el Pacífico Tropical Este, entre los $5^{\circ} 57^{\prime} \mathrm{S}$ y $18^{\circ} 47^{\prime} \mathrm{S}$ con una extensión aproximada de $1700 \mathrm{Km}$ desde la

${ }^{1}$ Laboratorio de Ecología Acuática. Facultad de Ciencias Biológicas. Universidad Nacional Mayor de San Marcos. Apartado 110058, Lima11, Perú.

E-mail Noemí Ochoa: aochoal@unmsm.edu.pe costa americana. Desde su descripción original no volvió a ser reportado en la literatura científica hasta el año 2000 cuando fue encontrado en el Golfo de México (HernándezBecerril y Bravo-Sierra, 2004), y luego fue observado en el 2002 en el área de Pisco, Perú (Sonia Sánchez, IMARPE, comunicación personal). En ambos casos con poquísimos ejemplares, 7 y 4 individuos, respectivamente.

Protoperidinium thulesense (Balech) Balech 1973 y Protoperidinium paulseni Abe 1981 son dos especies pequeñas que se reportan por primera vez para el Perú. Protoperidinium thulesense es típico del Pacífico sudamericano (Balech 1988) y Protoperidinium paulseni fue encontrado por primera vez en la localidad de Asamushi en el Pacífico del Japón.

\section{Material y Métodos}

El material fue obtenido dentro de los proyectos de investigación sobre comunidades marinas y medio ambiente que viene realizando la Universidad Nacional Mayor de San Marcos desde 1992. La colecta se realizó en 


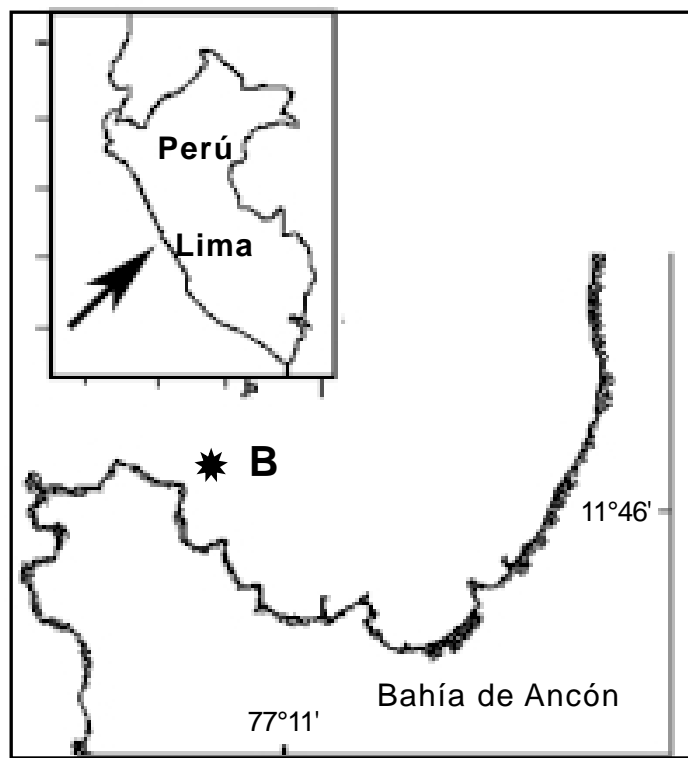

Figura 1. Bahía de Ancón. Ubicación de la estación B.

una estación fija, denominada Estación B, en la Bahía de Ancón ubicada en los $77^{\circ} 11^{\prime}$ W y $11^{\circ} 46^{\prime} \mathrm{S}$ (Fig. 1), durante el período 19922004. La bahía de Ancón es un área costera protegida y poco expuesta y la estación B tiene $15 \mathrm{~m}$ de profundidad.

Las muestras fueron colectadas de dos maneras, una con botellas Niskin a $0,7,5$ y 15 m de profundidad y otra con red estándar de fitoplancton de 75 m de tamaño de malla, mediante arrastres horizontales superficiales de 5 minutos a 3 nudos de velocidad. Las especies fueron identificadas con microscopía de luz.

\section{Resultados}

Orden DinOphysiales KofoID

Familia DinOphysiaceae Stein

GÉnero Dinofurcula KofoId y

SKOGSBERg 1928

\section{Dinofurcula cf. ventralis Kofoid y Skogsberg 1928}

Fig. 2 y 3

Sinonimia: Phalacroma Stein partim (1906c,1907a)

Cuerpo molariforme en vista lateral con dos procesos posteriores largos. La epiteca es convexa, baja, pero sobresale de la aleta cingular. La hipoteca es alargada con el margen dorsal sigmoideo y el ventral marcadamente convexo en la parte anterior, es bifurcada con 2 procesos posteriores, el proceso ventral muy delgado de extremo redondeado en forma de cuerno y el dorsal mas ancho de forma triangular adelgazado en el extremo distal. El cingulum es ancho y

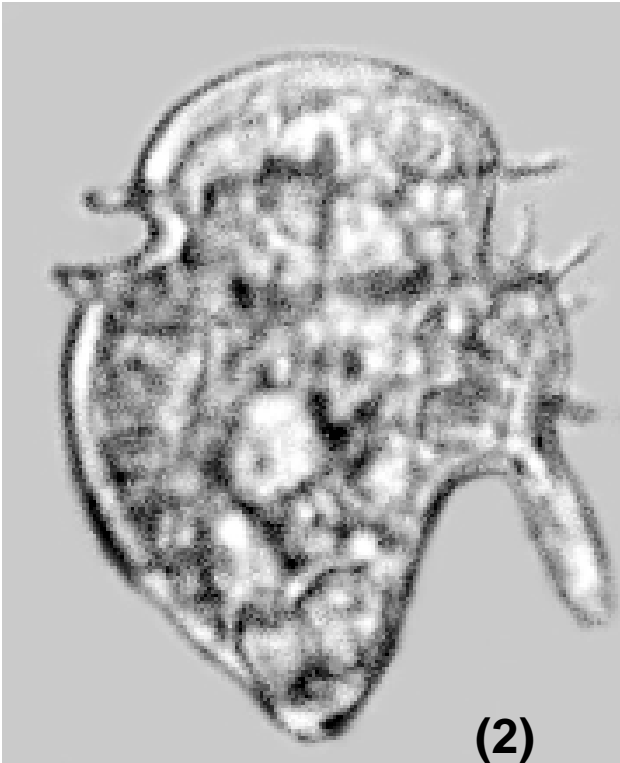

(2)

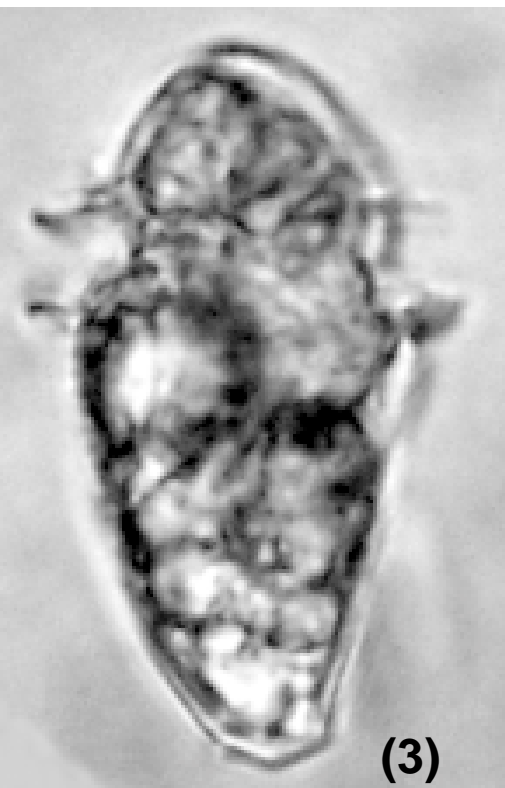

Figuras 2-3, Dinofurcula cf. ventralis; 2, Vista lateral izquierda; 3, Vista Dorsal. (Longitud $38,25 \mu \mathrm{m}$, profundidad $25,5 \mu \mathrm{m}$ ) 
excavado. No fue posible observar la posición del sulcus, pero por la posición de las aletas sulcales se asume que es ventral. Las aletas sulcales están reforzadas por 3 espinas (Fig. 2). La célula es fuertemente comprimida bilateralmente (Fig. 3).

Dimensiones: $38-41 \mathrm{~m}$ de longitud y 25-29 m de profundidad.

Distribución, ecología y taxonomía: Costa central del Perú, de 0 a $15 \mathrm{~m}$ de profundidad en un rango de 15 a $18,8^{\circ} \mathrm{C}$ de temperatura (Tabla 1).

Aún cuando no hemos observado el surco longitudinal, la posición de las aletas sulcales muestra que es ventral y no lateral como en

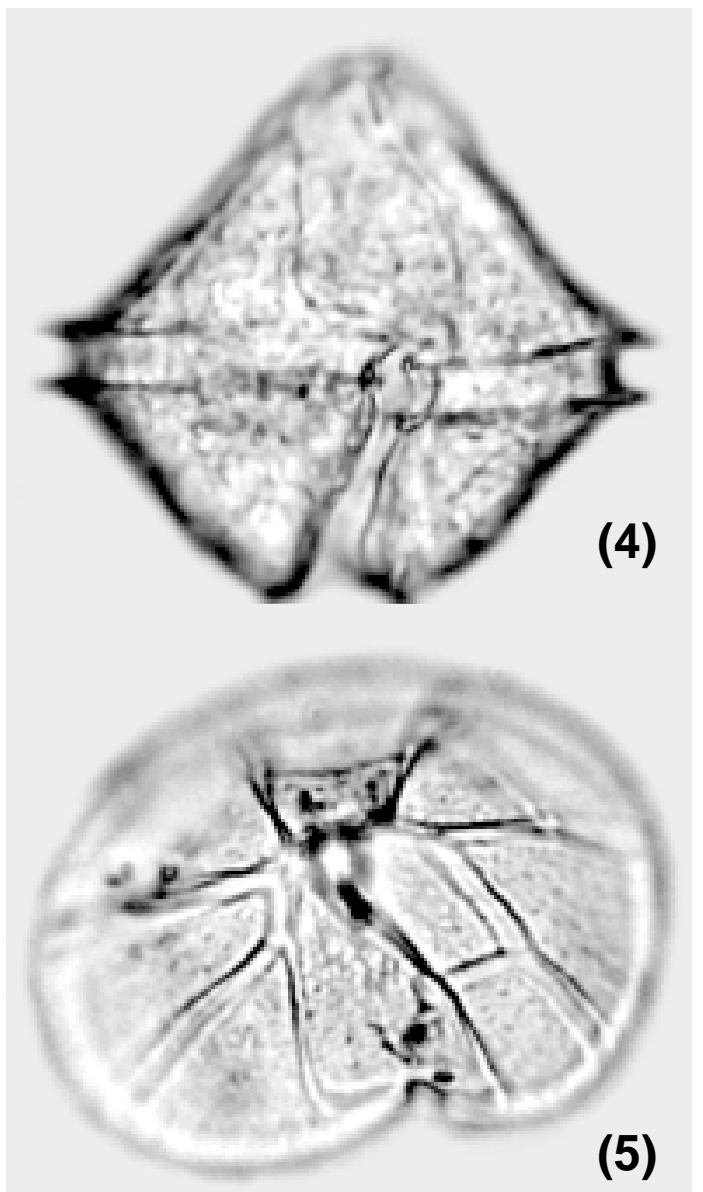

Figuras 4-5. Protoperidinium thulesense; 4, Vista ventral; 5, Placas epitecales. (longitud $61 \mu \mathrm{m}$, diametro transverso $68,8 \mu \mathrm{m}$, diametro dorso-ventral $53,5 \mu \mathrm{m}$.
Dinofurcula ultima por lo que hemos denominado a la especie como Dinofurcula cf. ventralis.

Orden Peridiniales Haeckel

Familia Peridiniaceae Ehrenberg

GÉnero Protoperidinium Bergh

Protoperidinium thulesense Balech 1973

Fig. $4-7$

\section{Sinonimia: Peridinium conicum f. Islandicum} Braarud, 1935.

Peridinium thulesense Balech, 1958.

Peridinium sympholis Hermosilla y Balech 1969.

Peridinium deficiens Abé 1981.

$P$. thulesense es una especie pequeña de forma pentagonal, epiteca cónica de lados planos o ligeramente cóncavos, patrón de placas peculiar en la mitad ventral de la epiteca debido a que la placa apical 4' es marcadamente reducida o está fusionada con la placa apical ventral (1'). Cingulum ancho, profundamente excavado, post ecuatorial, ligeramente ascendente. El sulcus es estrecho debajo del cingulum y ensanchado posteriormente, ocupando todo el ancho del extremo truncado. Hipoteca cónica trunca es casi 2/3 la longitud de la epiteca, excavada en su posición media ventral posterior, superficie de la teca finamente reticulada (Fig. 4).

Epiteca, dividida en 8 zonas meridionales por 8 suturas meridionales; excepto la que pasa por el borde izquierdo de la placa apical ventral 1', las otras convergen anteriormente para formar 2 grupos bilaterales, el izquierdo con 3 y el derecho con 4 ramas, o sea que sólo 3 suturas llegan hasta la canaleta apical. De las 3 zonas dorsales, dos están constituidas por 2 placas, una de ellas constituida por la placa intercalar $1^{a}$ y la precingular 3" y la otra por la placa intercalar $3^{\mathrm{a}}$ y la precingular 5"y una zona constituida por tres placas: apical 3', intercalar $2^{a}$ y precingular 4". De las 5 zonas ventrales, tres zonas están constituidas por una sola placa precingular 2", 6" y 7"; las otras 2 con 2 placas cada una, una conformada por las placas apical izquierda 2' y precingular 1" 

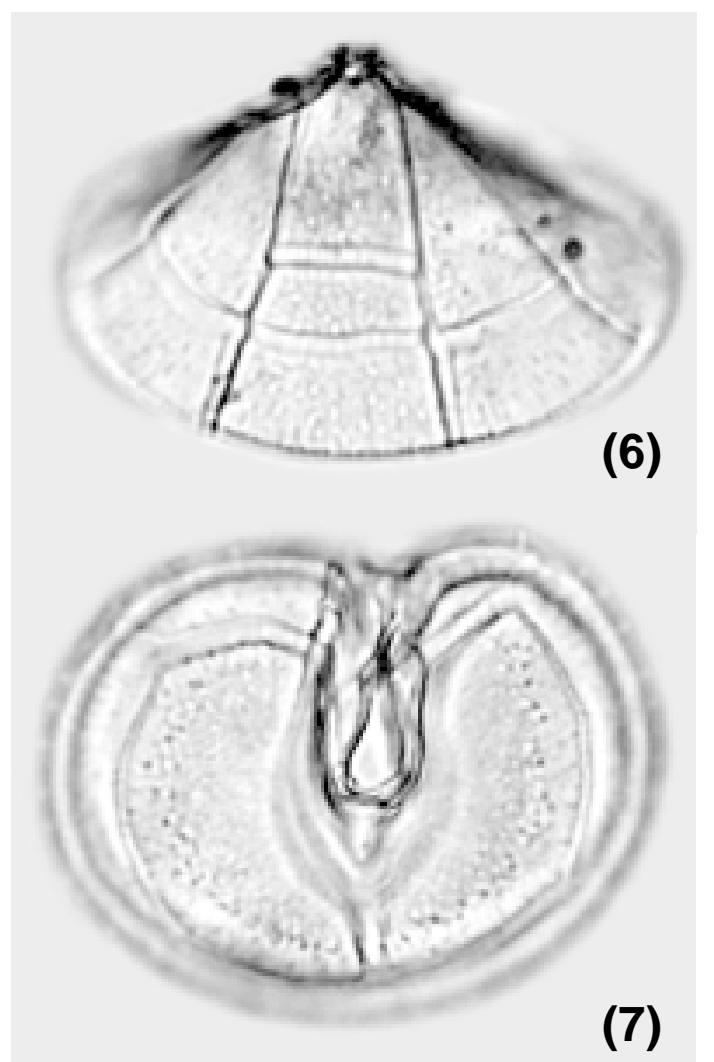

Figuras 6-7, Protoperidinium thulesense; 6, Epiteca vista dorsal; 7, Sección cingular de la hipoteca.

y la otra por las apicales 4' y 1', aunque generalmente éstas se fusionan completamente. Poro apical ligeramente ventral con hendidura o canaleta apical ventral extendiéndose oblicuamente hacia la izquierda (Fig. 5).

Las tres placas dorsales precingulares ( 3", 4" y 5") son casi iguales, ocupan la tercera parte de la epiteca. La intercalar izquierda $\left(1^{a}\right)$ es un poco más pequeña que la derecha $\left(3^{\mathrm{a}}\right)$. La apical dorsal (3') es pentagonal. La pared de la teca está cubierta con una malla poligonal fina (Fig. $6)$.

La sección cingular es reniforme y sus mitades bilaterales son subiguales la mitad izquierda es un poco más pequeña (Fig. 7).

Dimensiones: Longitud de 50-79 m, diámetro transverso de 51-77 m, diámetro dorsoventral de 43-64 m.

Distribución y ecología: Bahía de Ancón.
Encontrado en 11 oportunidades de agosto 1997 a diciembre 2003, entre 0 y $15 \mathrm{~m}$ de profundidad, en temperaturas de 14,5 a $20,2^{\circ} \mathrm{C}$ (Tabla 1 ).

Orden Peridiniales Haeckel

Familia Peridiniaceae Ehrenberg

GÉnero Protoperidinium Bergh

\section{Protoperidinium paulseni Abe 1981}

Fig. $8-13$

Sinonimia: Peridinium paulseni Abe, 1981

Peridinium subinerme Paulsen, 1931 (no 1908)

$P$. paulseni, es una especie pequeña, cíngulum circular, superficie de las placas cubierta con procesos diminutos que son mas prominentes en la hipoteca donde pueden crecer en longitud a manera de espinitas. La epiteca es baja, cónica. La hipoteca es tam-
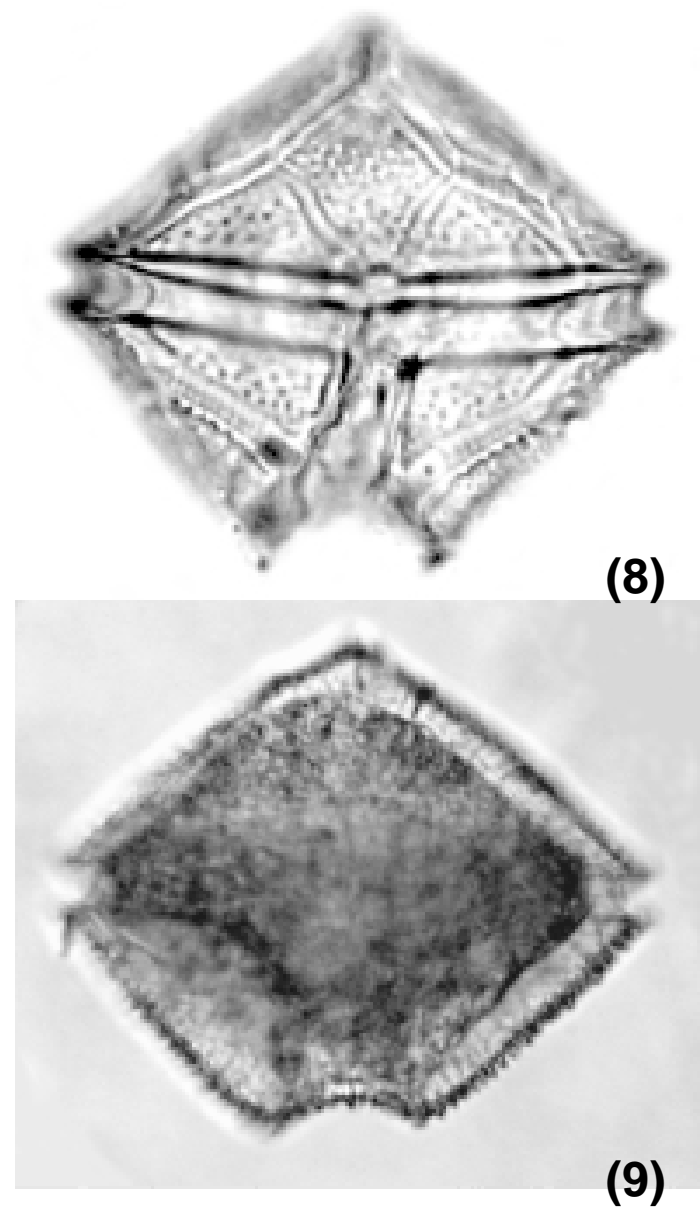

Figuras 8-9, Protoperidinium paulseni; 8, Vista ventral; 9, Vista dorsal. (Longitud 58,65 $\mu \mathrm{m}$, diametro transverso $71,4 \mu \mathrm{m})$ 
bién baja y presenta una depresión póstero media formada por la extensión dorsal del sulcus. El cingulum es ecuatorial, profundamente excavado con aletas hialinas bajas. Las dos placas cingulares ventrales corresponden en longitud con la longitud basal de las placas pre y post cingular (Fig. 8 y 9).

La sección cingular es reniforme con el lado ventral plano y dorsal ligeramente abultado. Placa precingular ventral izquierda (1") es un poquito mas pequeña que la ventral derecha (7"). Todas las placas precingulares son regularmente pequeñas en longitud cubriendo mas o menos la tercera parte de la epiteca. La apical dorsal (3') es pentagonal. Las tres dorsales centrales están desplazadas hacia la izquierda debido al desarrollo desigual de las
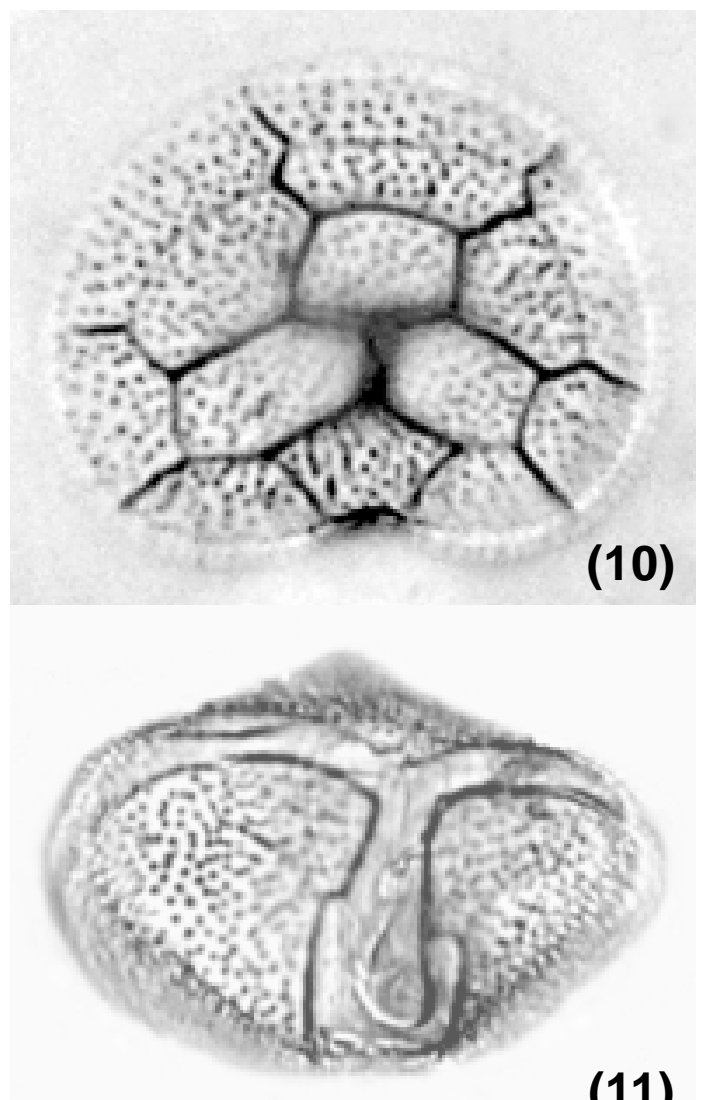

(11)

Figuras 10-11, Protoperidinium paulseni; 10, Vista apical; 11, Hipoteca, vista póstero ventral.

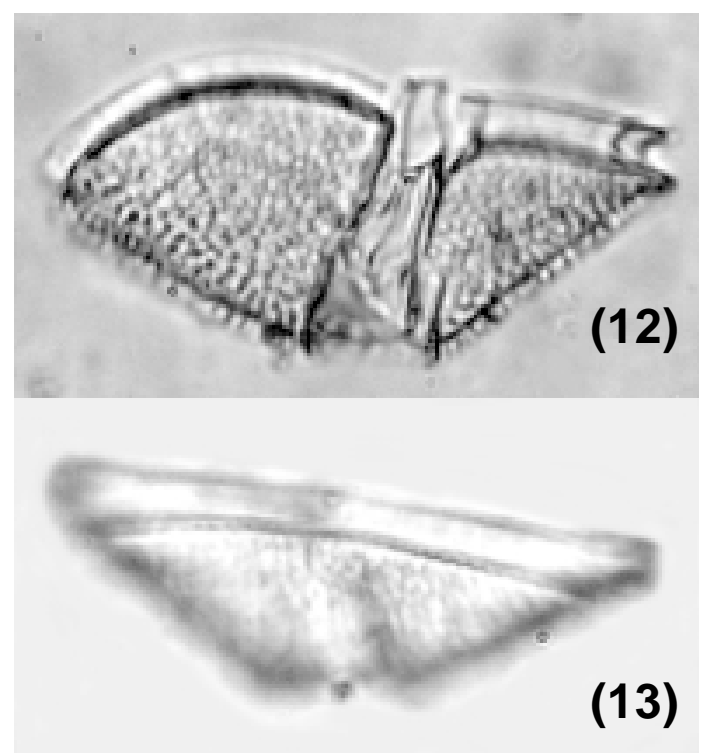

Figuras 12-13, Protoperidinium paulseni; 12 , Hipoteca, vista ventral mostrando las placas del cingulum (T, C2, C3); 13, Hipoteca, vista dorsal mostrando la placa del cingulum (C2).

placas bilaterales (Fig. 10).

En la hipoteca se observa el sulcus profundamente marcado que se extiende posteriormente hacia el centro de la misma excavando su región media antapical (Fig. 11).

Dimensiones: Longitud de 51-64 m, diámetro transverso 64-77 $\mathrm{m}$, diámetro dorsoventral 51-64 m.

Distribución, ecología y taxonomía: Bahía de Ancón. Fue encontrado en setiembre 2004 a 0 y 7,5 $\mathrm{m}$ de profundidad. En temperaturas de $14,6{ }^{\circ} \mathrm{C}$ a $14,8{ }^{\circ} \mathrm{C}$ (Tabla 1 ).

Es una especie muy parecida a Protoperidium punctulatum (Paulsen) Balech, 1974, del cual se diferencia por tener el transdiámetro claramente mas grande que el eje longitudinal, es hexa, el sulcus mas profundamente excavado, la placa $2^{\mathrm{a}} \tan$ ancha como 4" y todas las placas cubiertas con procesos diminutos que en la hipoteca crecen a manera de espinitas.

Esta especie fue descrita por Abé (1981) como Peridinium paulseni. Como posteriormente, no hemos encontrado ninguna refe- 
Tabla1. Relación de muestras donde se registraron Dinofurcula cf. ventralis, Protoperidinium thulesense y Protoperidinium paulseni

\begin{tabular}{lccccc}
\hline Fecha & Profundidad & Temperatura & D. cf. ventralis & P. thulesense & P. paulseni \\
\hline $31-08-97$ & 7,5 & 20,1 & & $X$ & \\
$28-09-97$ & 7,5 & 17,8 & & $X$ & \\
$18-10-97$ & 15 & 17,2 & & $X$ & \\
$15-01-00$ & 7,5 & 16,2 & & $X$ \\
$30-12-00$ & 7,5 & 14,4 & & $X$ \\
$30-12-00$ & 15 & 14,4 & & $X$ & \\
$15-03-02$ & 0 & 20,2 & & $X$ \\
$15-03-02$ & 15 & 19 & & $X$ & \\
$03-05-02$ & 7,5 & 17,4 & & $X$ & \\
$30-06-02$ & 7,5 & 15,2 & & $X$ & \\
$14-07-02$ & 0 & 15 & & & \\
$14-09-02$ & 0 & 14,8 & & & \\
$04-12-03$ & 0 & 17,2 & & & \\
$04-12-03$ & 15 & 15,5 & & & \\
$29-02-04$ & 7,5 & 18,79 & & \\
$30-09-04$ & 0 & 14,8 & & & \\
$30-09-04$ & 7,5 & 14,6 & & & \\
\hline
\end{tabular}

rencia que la adscriba al género Protoperidinium la estamos incluyendo dentro de este último género por presentar las características típicas del mismo, 3 placas cingulares y una placa transicional $\mathrm{o}$ «T» (Balech, 1974, 1988; Steidinger, \& Tangen, 1996) (Fig. 12 y 13).

\section{Agradecimientos}

Agradecemos el apoyo financiero del Fondo de Desarrollo Universitario (FEDU) de la Universidad Nacional Mayor de San Marcos. A los miembros del grupo DePSEA de la Facultad de Ciencias Biológicas, UNMSM, por el esfuerzo realizado durante la colecta del material biológico.

\section{Literatura citada}

Abe, T. H. 1981. Studies on the Family Peridinidae an Unfinished Monograph of the Armoured Dinofalgellate. Publications of the Seto Marine Biological Laboratory Epecial Publications Series. 6: 409 pp.
Balech, E. 1973. Segunda contribución al conocimiento del microplancton del mar de Bellingshausen. Contr. Inst. Antártico Argentino, 107, 63 pp.

Balech, E. 1974. El Género Protoperidinium Bergh, 1881 (Peridinium Ehr, 1831, partim. Rev. Mus. Argent. C. Nat., Hidrobiología, 4(I):1-79.

Balech, E. 1988. Los dinoflagelados del Atlántico Sudoccidental. Pub. Espec. Inst. Español de Oceanogr. N.1. Madrid, España. 219 pp y 88 láminas.

Hermosilla, J. y E. Balech. 1969. Un interesante Peridinium de tabulación anormal. Neotropica, 15(46):9-13

Hernández-Becerril, D. U. \& E. Bravo-Sierra. 2004. Observations on a rare planktonic dinoflagellate, Dinofurcula cf. ultima (Dinophyceae), from the Mexican Pacific. Phycologia, 43 (4): 341-345.

Kofoid, C. A. \& T. Skogsberg. 1928. The Dinoflagellata: The dinophysoidae. Memoirs of the Museum of Comparative Zoology at Harvard College 51: $1-766$.

Ochoa, N., O. Gómez, S. Sánchez y E. Delgado. 1999. Diversidad de diatomeas y dinoflagelados marinos del Perú. Bol. Inst. Mar Perú, 18(1-2):1-13.

Steidinger, . A. \& K. Tangen, 1996. Dinoflagellates. En: Identifying Marine Diatoms and Dinoflagellates. Carmelo R. Tomas (ed), Academic Press, San Diego, CA, USA, : 387-584. 